\title{
Recommendation System Based on the Learners' Tracks in an Intelligent Tutoring System
}

\author{
Mohammed Kamal Rtili, Ali Dahmani, and Mohamed Khaldi
}

\begin{abstract}
Internet has become the primary source of information, that's why data sources double each year and users are faced with a large number of irrelevant answers returned by classical information retrieval tools. During the last ten years, recommendation systems have emerged as an effective means to reduce the complexity in the search for information, these recommendation systems are based on the learner profile.

The learner profile is an important concept in the field of learning. Effective management of the learner profile is a good condition for better resource referral services.

The objective of our research is to develop a recommendation system by classifying learners by their behavior to provide them with resources according to their needs.
\end{abstract}

Index Terms - Intelligent tutoring systems, learning model, multi-agent system, recommendation system.

\section{INTRODUCTION}

The applications of artificial intelligence techniques in education have given rise to a number of education systems. Among the most frequently used, include among others, the intelligent tutoring systems. The latter allows the customization and adaptation of education content according to the learner profile, which improves the learning state of the learner. In fact, learners always have difficulties finding resources suitable to their field of study among the large amount of resources available, and the objective of this research is to incorporate mechanisms which use learners' behavior and a multi-agent technology to enable an easier access to the information they require: those that meet their expectations and tastes. The integration of these mechanisms will therefore increase learner satisfaction. Recommender systems are an example of such a mechanism, they provide users with personalized recommendations of resources by exploiting the knowledge that the system has on the users [1], or their past actions, in order to infer information according to their tastes and expectations. In sections 2 and 3, we introduce the intelligent tutoring systems as well as the recommendation systems. Then we

Manuscript received July 25, 2013; revised September 30, 2013. This is a thesis in computer science that takes place within IT, operations research and applied statistics laboratory at the Faculty of Sciences of Tetouan, Morocco. Its goal is to design and development a recommender system for an intelligent tutoring system which is based on the learner's trace to help the latter to access resources adapted to their needs easily.

Rtili Mohammed Kamal is with the LIROSA lab, Abdelmalek Essaadi University, Faculty of Sciences, av jbel oukaimden, res chaouen $4 \mathrm{~N}^{\circ} 3$, Tetouan, Morocco (e-mail: Rtili.kamal@gmail.com).

Dahmani Ali are with the LIROSA lab, Abdelmalek Essaadi University, Faculty of Sciences, B.P.2121, Mhanech II, Tetouan, Morocco (e-mail: alidahmani@hotmail.com,medkhaldi@yahoo.fr). present the learning model followed by the architecture of our system based on this model. Finally, we discuss the main contributions of this work, as well as the perspectives considered.

\section{INTELLIGENT TUTORING SYSTEMS}

The appearance of artificial intelligence techniques gave hope to respond to the critical of the EAO, especially the capacity to solve problems and explain solutions. The domain has therefore refocused around the theme of Education Intelligently Computer Aided, in particular Intelligent Tutoring Systems (ITS).

Intelligent Tutoring Systems (ITS) form a particular current of EIAO whose principle is the individualization in learning. The idea is to introduce a system that pays attention to the specific needs of the learner, evaluate and diagnostic his problems, and provides the necessary support. Thus, ITS respond to the need to place the learner "in the center of the learning situation."

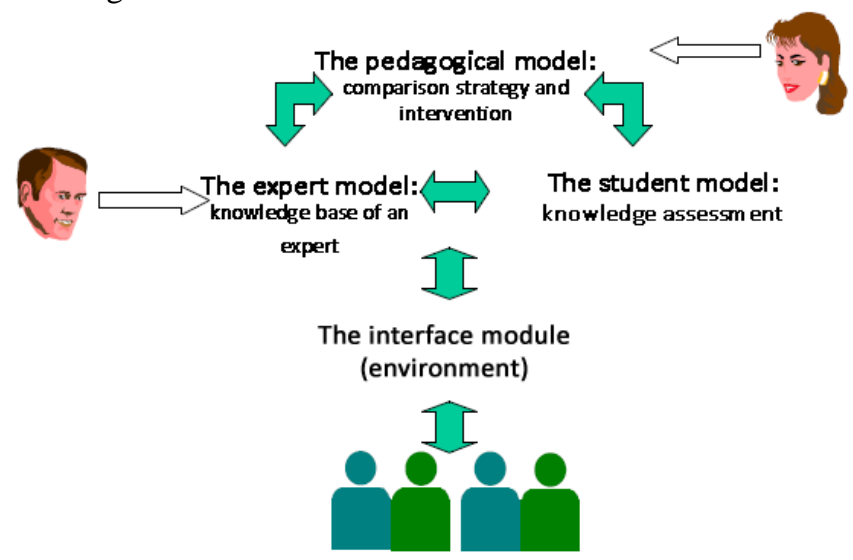

Fig. 1. Components of a system intelligent tutoring

An ITS should possess knowledge of various types: on the subject being taught, on how to teach, on the learner himself, and on how to communicate. This is why an intelligent tutoring system is generally composed of four modules, namely, the expert model, the pedagogical model, the student model and the interface [2] (see Fig. 1).

- The expert model offers an ITS the possibility to act as an expert, meaning that it's competent in the subject he teaches. This competence allows the tutorial to explain its reasoning and justify its decisions. To do so, an ITS should contain, in general, the specific knowledge on the domain being taught, as well as the reasoning process involved when solving problems.

- The pedagogical model is a set of specifications on how the system should build its interventions [3] This module 
interacts with the student more or less directly by selecting problems that must be solved, by guiding him to the solution, criticizing his performance, providing appropriate assistance when the student asked, and showing examples. To manage the interactions, the module also relies on knowledge about teaching strategies used by the human tutors, such as learning by example, learning by analogy, etc.

- The student model is a set of information about the state of knowledge, exact or erroneous, of the learner. Its construction was mainly established by comparison with the knowledge of the expert. The student model is also used to construct a diagnostic that can be used in ITS to make a decision of didactic nature (questions, explanations, remediation ...) [4]. In ITSs, the state of knowledge of the student is usually represented as a subset of the knowledge of the expert module. Therefore, the student model is constructed by comparing the performance of the learner with that of the expert has produced in the same circumstances. [5]

- The interface module; it is at this module that the interaction between the student and the ITS have become dynamic [6]. Its main role is to facilitate the communication of information between the system and the student. It must provide the student with activities through which he may acquire or consolidate concepts and approaches.

\section{RECOMMENDATION SYSTEM}

Recommender systems are software tools and techniques, providing suggestions of items to a user [7], [8].

RS have proven in recent years that they are a good way to deal with the problem of cognitive overload (Information Overload).

According to [7] two approaches are defined for RS:

- Approach based on the content: The recommendation based on the content is to analyze the content of resources or the descriptions of those resources in order to determine what resources are likely to be useful or interesting for a given user.

- Collaborative filtering: Collaborative filtering compares users with each other based on their past judgments to create communities, and each user receives documents deemed relevant by his community.

\section{THE USE OF THE LEARNER TRACE IN AN ITS TO CREATE MODELS THAT REGROUPS LEARNERS}

\section{A. Introduction}

Our system issues recommendations based on the individual needs of each learner. And the study of user behavior is a very important step in our work.

For each learner, we collect all the traces left by the learner; Jermann [9] defines a digital trail as a set of observations on the interaction of the learner with a system for analysis.

These traces are useful for customizing due they describe the behavior of users. Thus, we can use them in the modeling of learner's profiles by applying data mining techniques. The models obtained following this model are operational knowledge for the recommendation of resources.

\section{B. Trace Processing}

Following the study conducted by Manel ben sassi [10], processing models of the trace follow the same process of treatment to reach a track interpreted in different formats (Fig. 2).

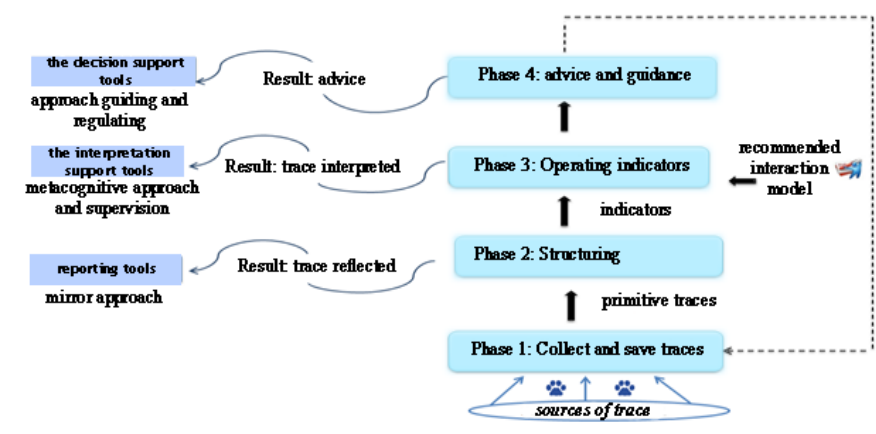

Fig. 2. Different phases of processing of the trace

\section{Learner Modeling}

\section{1) Introduction}

The learner model is a data structure that characterizes the knowledge acquired by the learner. According to Brusilovky [11], there are five main characteristics represented in the user model:

- The learner objective

- His Knowledge

- His education

- His experiences

- His preferences

This model allows an environment to adapt to each learner according to the information which he provides.

According to [11] there are several objectives of the learner modeling:

- Help a user during his education

- Adapt information, interface and support to the user.

- Facilitate the search for information.

- Provide the user with feedback reflecting his educational course

\section{2) Standards of learner model}

In order to offer the possibility of data exchange between systems, researchers have focused on the standardization of the learner's data. These efforts have given rise to the 2 standard PAPI and IMS LIP.

PAPI (Public and Private Information for Learners) model is a standard developed within the Learner Model Working Group [12].

The IMS LIP model (IMS Learner Information Package) is defined according to a XML structure for the purpose of exchanging the learner data between multiple systems especially learning management systems [12]. This model is based on the PAPI standard and has the necessary characteristics of a user for general uses.

These two standards classify information according to categories, to ensure the sharing of information between different systems. 
TABLE I: CATEGORIES OF INFORMATION FROM THE TWO STANDARD MODELS

\begin{tabular}{lll}
\hline \hline & PAPI Model & \multicolumn{1}{c}{ LMS LIP Model } \\
\hline & & + Identification information \\
& + Information on the learner's \\
+ Learner's & preferences \\
personal & + information on qualifications, \\
information & certifications, diplomas awarded to \\
+ Relational & the leaner \\
information & + Information activities related to \\
+ Security & work and education of the learner \\
information & + Information on the objectives of \\
+ Information on & the learner. \\
skills & + Information on skills \\
+ Portfolio & + Information about the learner's \\
information & hobbies \\
+ Learner & + Information on the content of the \\
preferences & learner's training \\
informations & + Relational information \\
& + Security information \\
& + Information on the organization \\
& associated to the learner
\end{tabular}

\section{DESIGN OF OUR RECOMMENDER SYSTEM BASED ON A MULTI-AGENT SYSTEM}

To increase learner satisfaction in an ITS, we thought of integrating a system whose role is to recommend appropriate resources for each learner after being assigned to a base profile that we have already defined in our system as the Fig. 3 show.

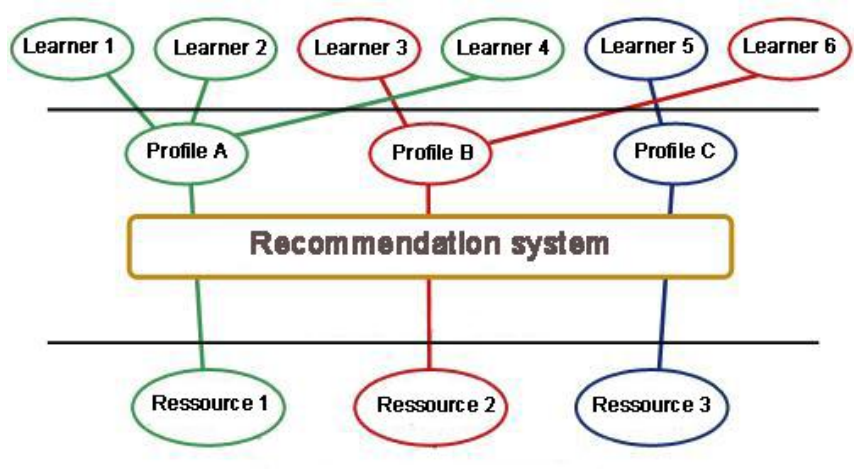

Fig. 3. Recommendation process learners

Our system will be based on a hybrid approach recommendation in which we will combine technical recommendation based on the content with collaborative filtering,k meaning, we will recommend resources that are based primarily on the comparison of these with the learner profile, and secondly on the opinions collected from other users (with similar interests) on their level of satisfaction after using the resources we have suggested to them.

The proposed system consists of the following steps:

- The collection of data based on the behavior of the learner: data are collected from their navigation on the training platform, results responses on tests, personal data, etc.

- Data processing: once the data is collected, they are cleaned and treated in order to prepare them as inputs to methods of data mining.

- Construction behavioral patterns of learners: in this step we apply the techniques of data mining on the preprocessed data (clustering, association rules).At the end of this step we obtain behavioral patterns and common interests on the groups of users.

- The recommendation of resources: This is the final step of our system where appropriate resources are recommended for each model that we have already built in the previous step. To achieve the above-mentioned steps, we will create a system with multiple modules each of which has its own task.

Presentation and description of the modules of the proposed system:

Our system for the recommendation of resources consists of four modules:

- The extraction module and information processing on the behavior of the learner: is the module responsible for collecting and processing the learner's traces. Once the data is collected, this module structured it according to a specific model to send it next to the data mining module.

- Data Mining module: it's the main module, it is critical for the actions of customization to undertake. It's based on individual profiles in order to analyze and classify those to generic profiles models, and deduce rules... All knowledge from this module will be stored in the knowledge base.

- The analysis and evaluation module: it retrieves knowledge from data mining to assess their relevance and ability to contribute to the recommendation.

- The recommendation module: it's module responsible for recommending resources to the appropriate profile.

Each module is responsible for the realization of a very special phase of the recommendation process. In each phase one or more agents are responsible for the realization of the objective of this phase. The extraction module and information processing on the behavior of the learner uses agents that collect, process and structure data. After the data mining module requests two agents, each of which encapsulate a method of search namely clustering agent and association rules agent, to produce thereafter behaviors models, which will subsequently be exploitable by the recommendation module consists of the recommendation agent. This Fig. 4 shows the architecture of such system:

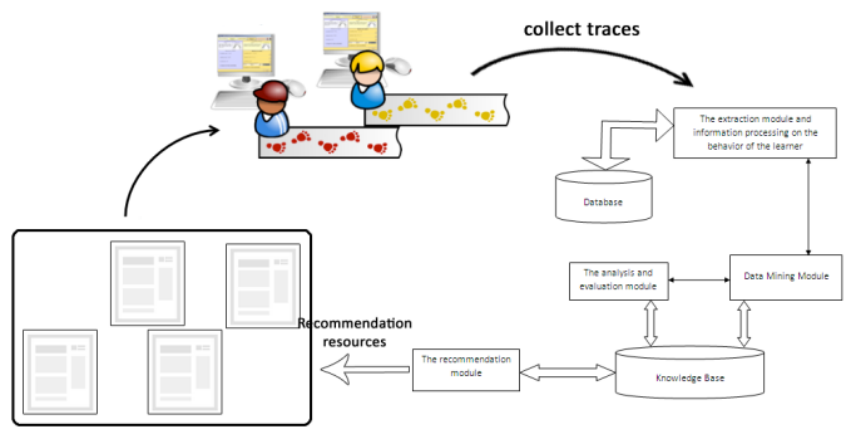

Fig. 4. architecture of our recommendation system

The objective of our system is the discovery of operational knowledge to automate the recommendation process. To achieve a goal, the agents cooperate and coordinate through messages. These messages are informational messages sent either to activate other agents, or to give information. 


\section{CONCLUSION AND PERSPECTIVES}

We have outlined the purpose of the integration of a recommendation system in an intelligent tutoring system.

We introduced the principle and components of an intelligent tutoring system, and the use of our recommendation system in the latter.

We consider focusing our future research to determine firstly the data needed to create a user profile. And then we will determine the agents of each phase and the role of each to give the final architecture of our recommendation system.

\section{REFERENCES}

[1] R. Burke, K. Hammond, and E. Cooper, "Knowledgebased navigation of complex information spaces," in Proc. of the 13th National Conference on Artificial Intelligence (AAAI'96), 1996, pp. 462-468.

[2] J. F. Nicaud and M. Vivet, Les tuteurs intelligents, réalisations et tendances de recherche, 1988.

[3] J. Self, "Students Models: What Use Are They?," in Proc. workshop IFIP/TC3, 1987, pp. 73-85.

[4] J. Self, "Cognitive diagnosis for tutoring systems," in Proc. 10th European Conference On Artificial Intelligence, ECAI92, 1992, pp. 699-703.

[5] I. P. Goldstein, "The genetic graph: a representation for the evaluation of procedural knowledge," in Intelligent Tutoring Systems, D. Sleeman and J. S. Brown, Eds. Academic Press, New York, 1982, pp. 51-78.

[6] M. J. Baker and K. Lund, "Promoting reflective interactions in a computer-supported collaborative learning environment," Journal of Computer Assisted Learning, vol. 13, pp. 175-193, 1997.

[7] R. Burke, Hybrid web Recommender Systems, pp. 377-408, 2007.

[8] T. Mahmood and F. Ricci, "Improving recommender systems with adaptive conversational strategies," in Proc. 20th ACM conference on Hypertext and hypermedia HT '09, 2009, pp. 73-82.

[9] P. Jermann, A. Soller, and M. Muehlenbrock, "From mirroring to guiding: A review of state of the art technology for supporting collaborative learning," in Proc. the First European Conference on Computer-Supported Collaborative Learning, 2001, pp. 324-331.

[10] M. Ben Sassi and M. Laroussi, Towards Learners' Tracks Standardisation, 2012.

[11] P. Brusilovsky, "Methods and technique of adaptive hypermedia," User Modeling and User-Adapted Interaction, vol. 6, 1996, pp. 87-126.

[12] CEN. (2009). Learning Technologies Standards Observatory. [Online]. Available: http://Www.Cen-Ltso.Net

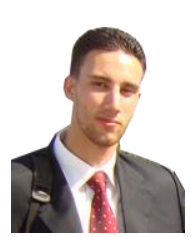

Rtili Mohammed Kamal is a $\mathrm{PhD}$ student in the Department of Computer Science, LIROSA Loboratory, Faculty of Sciences, Abdel Malek Essaadi University, Tetouan Morocco.

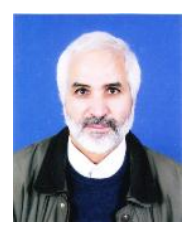

Dahmani Ali is a research professor in mathematics with Computer lab, operational research and applied statistics, Abdelmalek Essaadi University, Faculty of Sciences, Tetouan, Morocco

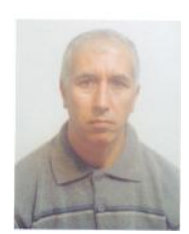

Khaldi Mohamed is a research professor in e-LEarning since 2008 with Computer lab, operational research and applied statistics, Abdelmalek Essaadi University, Faculty of Sciences, Tetouan, Morocco 